\title{
La "mariposa papagayo" de Molina (Insecta: Lepidoptera): Refutando la combinación Castnia psittacus (Molina, 1782)
}

\section{The "parrot-butterfly" of Molina (Insecta: Lepidoptera): Refuting the combination Castnia psittacus (Molina, 1782)}

\author{
Mario I. Ramos-González ${ }^{1,2, *}$, Carlos Zamora-Manzur ${ }^{3,4}$ \& Luis E. Parra ${ }^{1}$ \\ 1'Departamento de Zoología, Facultad de Ciencias Naturales y Oceanográficas, Universidad de Concepción, Casilla 160-C, \\ Concepción, Chile. \\ ${ }^{2}$ Programa de Magíster en Ciencias mención Zoología, Departamento de Zoología, Facultad de Ciencias Naturales y \\ Oceanográficas, Universidad de Concepción, Chile. \\ ${ }^{3}$ Departamento de Ecología, Facultad de Ciencias, Universidad Católica de la Santísima Concepción, Alonso de Rivera 2850, \\ Concepción, Chile. \\ ${ }^{4}$ Programa de Doctorado en Sistemática y Biodiversidad, Departamento de Zoología, Facultad de Ciencias Naturales y \\ Oceanográficas, Universidad de Concepción, Chile. \\ *Email: marioramos@udec.cl
}

\begin{abstract}
RESUMEN
La combinación Castnia psittacus (Molina) designada por Philippi (1867) y argumentada posteriormente por Ureta (1955) es invalidada por deficiencias, incoherencias, tergiversaciones y malas interpretaciones de la descripción realizada por Molina. Castnia eudesmia Gray stat. rev., es propuesta como la combinación válida, mientras el epíteto "psittacus" es asignado al único Papilionidae de Chile Battus polydamas.
\end{abstract}

\begin{abstract}
The combination Castnia psittacus (Molina) designated by Philippi (1867) and later argued by Ureta (1955) is invalidated by deficiencies, inconsistencies, misrepresentations and misinterpretations of the description made by Molina. Castnia eudesmia Gray stat. rev. is proposed as the valid combination, while the epithet "psittacus" is assigned to the only Papilionidae of Chile Battus polydamas.
\end{abstract}

La obra de Molina es, sin duda, una obra trascendental, pionera y de suma importancia en lo que respecta al estudio de la biodiversidad chilena. En ella se dan las primeras descripciones y nombres científicos de la biota distribuida en el territorio chileno, siguiendo la nomenclatura binomial y clasificación propuesta por Linneo (1758) (PérezD’Angelo 1966; Stuardo 2007). Sin embargo, algunas de las descripciones propuestas por Molina resultan ambiguas e inexactas, lo que sumado a la carencia de ilustraciones y material tipo, ha provocado que, de los 117 nombres específicos propuestos, sólo 53 sean válidos en la actualidad (sensu Stuardo 2007). Entre las especies dudosas que han causado confusión en la literatura se encuentran dos especies de lepidópteros chilenos: el heterócero Phalaena ceraria Molina, 1782 y la mariposa Papilio psittacus
Molina, 1782; siendo esta última la especie que más controversias ha suscitado (Pérez-D’Angelo 1966).

El heterócero diurno Castnia eudesmia Gray, 1838, conocido comúnmente como mariposa del chagual, es la única especie de Castniidae reportada para Chile (Parra \& Villagrán-Mella 2008). El ciclo biológico de la especie se encuentra bien documentado (e.g., Philippi 1863; Gazulla \& Ruiz 1928; Reed 1935; Angulo 1998; Niemeyer et al. 2002; Angulo \& Olivares 2009). Sin embargo, Moraes \& Duarte (2014) incluyeron esta especie en incertae sedis, debido a que estaría más cercana filogenéticamente a Ceretes Schaufuss que a Castnia Fabricius (probablemente ocurrió un error de identificación, el ejemplar utilizado fue recolectado en Ecuador y Castnia eudesmia es endémica de Chile). 
En la literatura, la mariposa del chagual ha sido frecuentemente mencionada bajo la combinación Castnia psittacus (Molina, 1782) (e.g., Ureta 1955; Angulo \& Olivares 1993; Angulo 1998), un nombre que surge del comentario hecho por Philippi (1867) donde indica que $P$. psittacus corresponde a $C$. eudesmia a partir de la corta e imprecisa descripción y diagnosis, en italiano y latín respectivamente, provista por Molina (1782) sobre esta especie, y traducida al español por Don Domingo Joseph como:

"Como la familia de las Orugas es en aquel Reyno tan sumamente variada, aparece por todas partes en la primavera un numero prodigioso de mariposas, entre las quales se hallan muchas sumamente admirables, no menos por su magnitud, que por la riqueza y brillo de sus coloridos. Hay particularmente una del primer órden, a la qual he puesto el nombre de Papagayo, Papilio psittacus*, por estar variamente pintada de todos aquellos colores que se admiran en los mas hermosos papagayos. La parte superior de la cabeza aparece pintada de un bello roxo de cinabrio manchado de amarillo; este color domina en toda la espalda, pero venado de verde, roxo, y azul celeste: las alas son por encima verdes, con manchas irregulares, amarillas y azules, y por debaxo son rubias: el vientre es celeste, salpicado de pardo y de gris; y las antenas, hechas á manera de mazas, son de color purpura. Hay otra del mismo tamaño, que los muchachos llaman Paloma, Papilio leucothea, la qual es enteramente de un color blanco argentado, a excepción de las antenas y de los pies, que son negros." (sic., Molina (1788)). Diagnosis en latín en el pie de página como “* Papilio N. Alis dentatis viressentibus, luteo, caeruleoque maculatis, subtus flavis".

Ureta (1955) realizó un estudio de la descripción original indicando que esta especie, según sus palabras, corresponde "indudablemente" al Castniidae chileno, por lo cual el nombre válido del taxón, siguiendo el principio de prioridad (Art. 23 del CINZ), sería C. psittacus (Molina, 1782) y no $C$. eudesmia siendo este nombre relegado a sinónimo junior. Posteriormente, gran parte de la literatura trata a esta especie bajo la combinación C. psittacus (Mol.). Ureta (1955) inició sus argumentos basado en lo expuesto por Philippi (1867), quien afirma que C. eudesmia "es la que Molina describe con el nombre de Papilio psittacus". Esta afirmación carece de argumentos válidos, y está basada en la tan criticada descripción original de Molina. Un escrutinio minucioso de la descripción original, diagnosis y las seis afirmaciones que Ureta (1955) emplea para validar la combinación $C$. psittacus, revela una serie de importantes problemas, como argumentos inconsistentes o sin importancia, tergiversaciones e incoherencias, que impiden corroborar su propuesta nomenclatural, los cuales son abordados individualmente a continuación.

Afirmaciones numeradas, extraídas de Ureta (1955), seguido de nuestras propuestas correspondientes a cada uno de sus argumentos:

1. "Aparece en la primavera", la hemos colectado desde los primeros días de noviembre.

Irrelevante. Un enorme número de lepidópteros, incluyendo especies chilenas, vuelan durante los meses de primavera (Benyamini et al. 2014; Pyrcz et al. 2016), por lo que el período de vuelo no es un rasgo suficientemente determinante ni adecuado para delimitar un taxón.

2. De acuerdo a la descripción original: "Entre las quales se hayan muchas sumamente admirables no menos por su magnitud, etc", el autor reconoce que es grande.

Engañoso. Molina (1782) indica que P. psittacus es del tamaño de Mathania leucothea (Mol.). Ureta indica que la expansión alar de los machos de $C$. eudesmia varía entre los $60-70 \mathrm{~mm}$ y las hembras miden hasta $100 \mathrm{~mm}$, mientras $M$. leucothea varía entre 44 y $62 \mathrm{~mm}$, siendo una cifra evidentemente menor a la del Castniidae chileno e interpretado erróneamente como coincidente por el autor. Esta incongruencia entre las envergaduras alares del Castniidae y M. leucothea, y por ende P. psittacus, ya había sido advertida por Reed (1935).

3. Es para Molina una de nuestras más bellas mariposas, concepto compartido por muchos de nosotros.

Evidencia inadecuada y subjetiva. El concepto de belleza corresponde únicamente a una apreciación personal y subjetiva, y no a un carácter diagnóstico para una especie. Enfatizar que esta opinión es compartida por muchos autores recae en una falacia ad populum.

4. "Tiene todos los colores de los más hermosos papagayos: verde, rojo, azul, celeste, amarillo, naranja, blanco, etc." (Molina, 1788)

Impreciso. Los colores y en general la maculación alar son caracteres morfológicos variables que por sí solos dificultan la delimitación de especies, por tanto, no es un buen argumento considerando que no es la única especie chilena con los colores listados por Molina. Por la palabra "etcétera", se deduce que la especie tendría más de siete colores, lo cual probablemente sea un rasgo irreal o una exageración del autor al no contar con ejemplares al momento de la descripción; exageraciones y malas descripciones de especies con rasgos inexistentes no están ausentes en la obra de Molina (Stuardo 2007). Por ejemplo: el cástnido chileno no presenta coloración verde sobre sus alas y ninguna mariposa chilena con el tamaño descrito posee un abdomen celeste salpicado 
de pardo y gris. Sin embargo, Battus polydamas archidamas (Boisduval, 1836) (Papilionidae), a diferencia de C. eudesmia, presenta la faz inferior de las alas amarillo-pálidas con manchas rojas, blancas y azules, además de reflejos azulados visibles en la región dorsal de las alas anteriores (en ejemplares frescos); también posee escamas verdes en la banda que cruza la faz superior de las alas posteriores. Por otro lado, Eumorpha labruscae (Linneo, 1758) (Sphingidae) también presenta los colores mencionados por Molina.

5. Según Ureta (1955), pertenece a las heteróceras, pues tiene "las antenas, hechas a modo de maza" (Molina, 1788)

Contradictorio e ilógico. Las antenas en forma de maza, contrario a lo expuesto por Ureta, son típicamente de Rhopalocera (i.e., género Papilio sensu Linneo) y no de Heterocera (i.e, Sphinx y Phalaena sensu Linneo). Si bien las antenas de Castniidae (así como de Uraniidae y Sematuridae) presentan antenas claviformes, la diagnosis presentada por Molina (1782) como "Papilio Nymphalis" es clara referencia a la morfología típica de Rhopalocera (Butler 1881).

6. Es la única mariposa chilena, y ponemos énfasis sobre este hecho, que tiene "la parte superior de la cabeza pintada de un bello rojo de cinabrio, manchado de amarillo; este color domina toda la espalda". Este color amarillo se debe al polen de las flores del chagual (Ureta, 1955).

Inconsistente. Si bien el polen de las flores del chagual puede llegar a teñir el color de la cabeza de la mariposa, este color nunca domina por sobre el color oscuro del tórax y abdomen como indica Molina en la descripción. Los rasgos descritos son congruentes con la especie $B$. polydamas archidamas donde se aprecian en las patagias, tórax y abdomen numerosas escamas amarillo-anaranjadas.

Adicionalmente a lo expuesto arriba, la diagnosis de $P$. psittacus en latín entrega información que no fue considerada por Ureta (1955): "Alis dentatis virescentibus, luteo, caeruleoque maculatis, subtus flavis", es decir, alas en la región dorsal con un color verdoso con maculación amarillenta y azulada, su faz ventral es amarilla, además de tener bordes externos dentados. Todas las características anteriores están ausentes en el Castniidae, y dentro de las mariposas chilenas estos caracteres son congruentes con $B$. polydamas archidamas.

Butler (1881) fue el primero en dar cuenta de lo dudosa de la descripción de Molina (1782), comentando que no concuerda con ninguna de las especies chilenas y la ubica, con dudas, entre las sinonimias de Papilio bias. Herrera (1954) reconoce a la especie descrita por Molina (1782) como P. (Battus) psittacus (Mol.), la mariposa de la oreja de zorro. Aunque posteriormente Herrera \& Etcherverry (1960), indican que el epíteto específico "psittacus" Molina causa incertidumbre para algunos autores, dividiendo las opiniones en tres posturas: a) quienes la consideran sinónimo de $B$. polydamas archidamas debido a la congruencia de sus rasgos con la diagnosis en latín; b) quienes la consideran "nom. incert."; y c) quienes se lo adjudican a otra mariposa chilena como Castnia siguiendo la opinión de Philippi (1867) y los argumentos de Ureta (1955). Posteriormente, Pérez-D'Angello (1966) analiza los nombres de los insectos propuestos por Molina (1782) y concluye que el epíteto psittacus podría corresponder tanto a Castnia como a Battus. Finalmente, Herrera \& Atria (1992) proponen que el epíteto psittacus Mol. debería ser invalidado para evitar mayores controversias, no pudiendo ser aplicado a ninguna mariposa chilena. A pesar de ello, el uso del epíteto específico "psittacus" Molina persistió.

Por otra parte, Reed (1935) sugiere a C. eudesmia como la combinación válida para referirse al Castniidae, indicando que la propuesta de Philippi es errónea, notando la incongruencia de tamaños entre la mariposa descrita por Molina y el cástnido. Sin embargo, Ureta (1955) al validar la combinación C. psittacus propuesta por Philippi, indica que Reed (1935) pensaría de manera semejante a él e incluso habría instado a publicar dicho trabajo, lo que contradice su sugerencia previa. Sin embargo, más adelante Miller (1995) deja fuera de los Castniidae Neotropicales a la especie "psittacus" de Molina, utilizando en cambio la combinación Castnia eudesmia para referirse al único cástnido chileno. Posteriormente, Lamas (1995) realiza una revisión de la lista de Castniidae dada por Miller (1995) para el Neotrópico, proponiendo nuevos arreglos taxonómicos y reduciendo el número de especies válidas de 134 a 81 . En este listado se encuentra nuevamente C. eudesmia Gray, entre cuyas sinonimias no incluye a $C$. psittacus (Mol.). Más adelante, González et al. (2010) y Penco (2011) comentan que $C$. eudesmia Gray es el nombre correcto para denominar a la Castnia de Chile y que $C$. psittacus (Mol.) es un nombre propuesto por Philippi nacido de un error de interpretación, el cual desafortunadamente fue ampliamente aceptado y propagado perpetuándose hasta la actualidad. Sin embargo, en ninguno de los trabajos posteriores a Ureta (1955) se ha revalidado formalmente la combinación $C$. eudesmia ni se ha presentado evidencia en contra de la combinación C. psittacus.

Respecto al epíteto "psittacus", Rothschild \& Jordan (1906) proponen la sinonimia de P. psittacus y $P$. archidamas basándose en los caracteres mencionados por Molina (1782) en la diagnosis de la especie, sin embargo, menciona a "psittacus" como sinónimo junior de “archidamas". Posteriormente, Tyler et al. (1994) continúan con esta propuesta mencionando dentro de los papiliónidos americanos a Battus polydamas psittacus (Molina, 1782), refiriéndose al sinónimo senior de la subespecie de $B$. polydamas (Linnaeus, 1758) distribuida en Chile. Esta 
combinación ha sido seguida por otros autores posteriores (e.g., Lamas 2004; Penco 2011; Penco \& Di Iorio 2014), sin embargo, este arreglo taxonómico ha pasado inadvertido en la literatura entomológica chilena y debido a los argumentos proporcionados por Ureta (1955) los nombres Castnia psittacus y $B$. polydamas archidamas son aún utilizados para referirse a ambos taxa (e.g., Peña \& Ugarte 2006; Benyamini et al. 2014).

Finalmente, la evidencia presentada nos permite refutar la propuesta realizada por Philippi (1867) y validada por Ureta (1955). Por lo tanto, establecemos a Castnia eudesmia
Gray stat. rev. como un taxón válido e independiente de la especie "psittacus" de Molina. Por otro lado, basándonos en la descripción y tomando en cuenta la diagnosis en latín presentada por el abate Molina, consideramos a Battus polydamas psittacus (Molina) nom. dub. (Papilionidae) como combinación válida. Para subsanar la carencia del tipo portanombre de Molina, en este trabajo designamos como Neotipo (Art. 75 del CINZ) para la especie Papilio psittacus Molina, 1782 a: 1 Chile, Talagante 10-XII-1964 Leg. J. Herrera (depositado en la colección del MZUC-UCCC) (Fig. $1 \mathrm{c}-\mathrm{d}$ ).

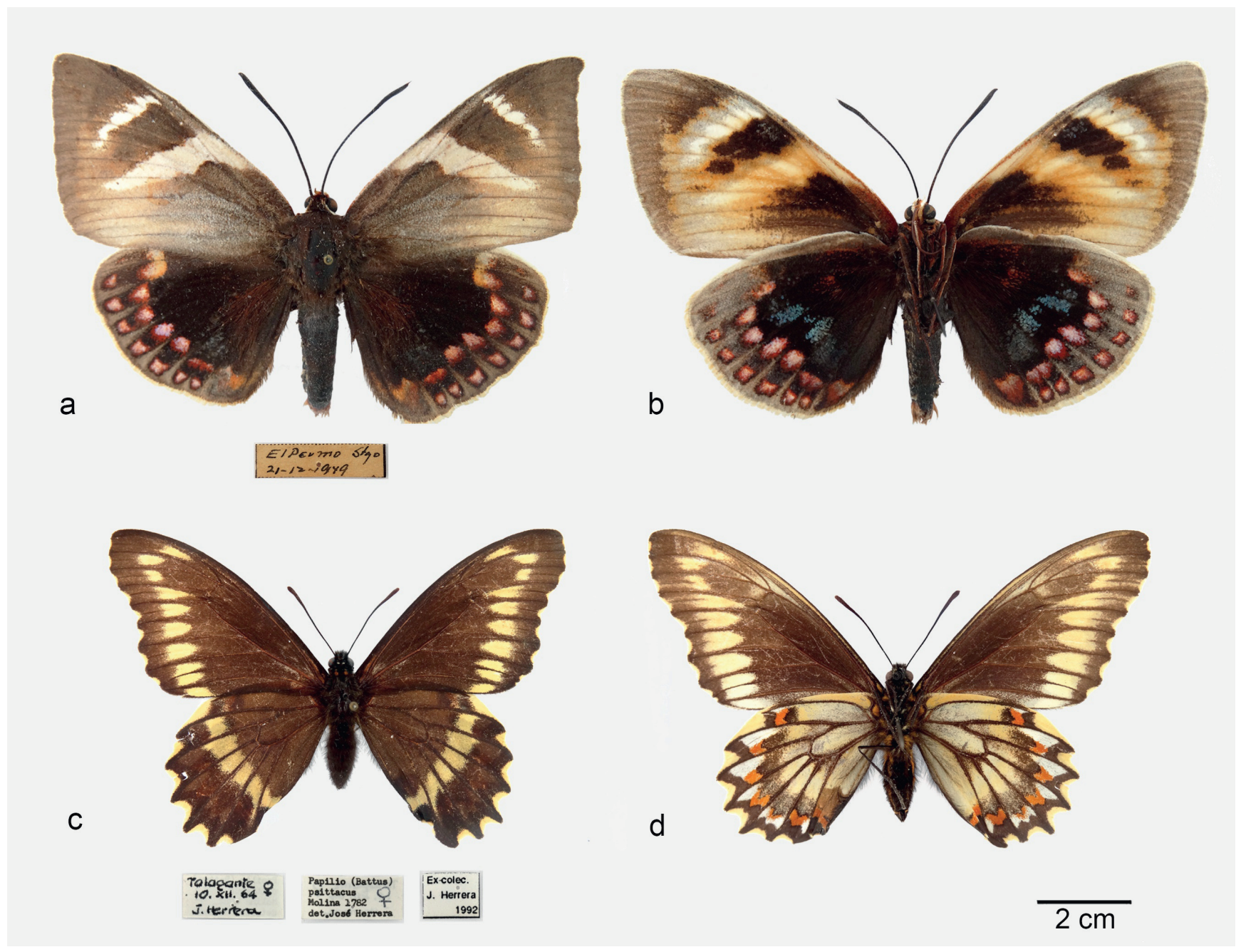

Figura 1. Ejemplares adultos de Castnia eudesmia Gray y Battus polydamas psittacus (Mol.). a) macho de Castnia eudesmia en vista dorsal y b) en vista ventral, c) Neotipo de Battus polydamas psittacus en vista dorsal y d) en vista ventral. / Adult specimens of Castnia eudesmia Gray and Battus polydamas psittacus (Mol.). a) male of Castnia eudesmia in dorsal view and b) ventral view c) Neotype of Battus polydamas psittacus in dorsal view and d) ventral view. 


\section{Nombre válido para la mariposa del chagual}

CAstnildae

Castnia eudesmia Gray, 1838 stat. rev. (Fig. 1 a-b) Castnia eudesmia Gray, 1838. Gray, 1838: 145; HerrichSchäffer, 1854: 140, 141; Walker, 1854: 19; Blanchard, 1852: 47, lám. 5, fig. 8; Philippi, 1863: 337; Boisduval, 1875: 517; Westwood, 1877: 177; Butler, 1877: 3, lám. 1, fig. 2; Butler, 1882: 4; Crowley, 1884: i; Kirby, 1897: 36, lám. 72, fig.1; Houlbert, 1918: 340, figs. 15,16; Gazulla \& Ruiz 1928: 290, fig. 48 (autoría errónea como C. eudesmia Blanchard); Reed, 1935: 267, fig. 42; Miller, 1995: 135; Lamas, 1995: 79; Angulo \& Olivares, 2009: 56, figs. 2-12; González et al., 2010: 394; Penco, 2011: 28; Moraes \& Duarte, 2014: 391.

Castnia eudesmia omissa Pfeiffer, 1915. Pfeiffer, 1915: 5, figs. 1-4; Miller, 1995: 135.

Castnia eudesmia var. chilena Houlbert, 1918. Houlbert, 1918: 341.

Elina eudesmia (Gray, 1838). Houlbert, 1918: 686; Latty, 1922: 78 [Elina Houlbert, 1918; homónimo junior de Elina Blanchard, 1852]

Castnia psittacus (sensu Auctorum) (nom. nud.) [error de identificación de Papilio psittacus Molina (Papilionidae)] Philippi, 1867: 779; Ureta, 1955: 229; Angulo, 1998: 211 syn. nov.

\section{Nombre válido de la mariposa papagayo "psittacus"}

\section{Papilionidae}

Battus polydamas psittacus (Molina, 1782) (Fig. $1 \mathrm{c}-\mathrm{d}$ ) Papilio psittacus Molina, 1782: 211 [nom. dub.] Papilio bias Roger, 1826: 159; Butler, 1881: 474; Bartlett-Calvert, 1886: 40; Bartlett-Calvert, 1898: 97; Elwes, 1903: 293; Silva-Figueroa, 1920: 212, fig. 64-65; Ureta, 1939: 256 fig. 5.

Papilio lindeni Ehrmann, 1925: 90

Papilio archidamas Boisduval, 1836: 321; Feisthamel, 1839: 37 Blanchard, 1852: 8, lám 1, fig. 1 a,b; Reed, 1877: 653; Rothschild \& Jordan, 1906: 516; Ureta, 1937: 122.

Battus archidamas (Boisduval, 1836). Pérez-D’Angelo, 1965: 4

Battus polydamas archidamas (Boisduval, 1836). Peña \& Ugarte, 2006: 188; Benyamini et al., 2014: 12 Battus polydamas psittacus (Molina, 1782). Tyler et al., 1994: 28; Lamas, 2004; Penco \& Di Iorio, 2014: 54.

\section{AGRADECIMIENTOS}

CZ-M agradece el apoyo financiero de la "Beca Doctorado
Nacional" CONICYT No 21161423 y al proyecto EDPG LPR-161 de la Dirección de Postgrado de la Universidad de Concepción. MR-G agradece el apoyo financiero de la "Beca de Magíster Nacional” CONICYT No 22162333.

\section{REFERENCIAS}

Angulo, A.O. 1998. El huevo de Castnia psittacus Molina (Lepidoptera: Castniidae). Gayana Zoología 62(2): 211213.

Angulo, A.O., Olivares, T.S. 2009. The Real Larva of Castnia eudesmia (Lepidoptera: Castniidae). Tropical Lepidoptera Research 19(1): 56-57.

Benyamini, D., Ugarte, A., Shapiro, A.M., Mielke, O., Pyrcz, T., BÁLINT, Z. 2014. An updated list of the Butterflies of Chile (Lepidoptera, Papilionoidea and Hesperioidea) including distribution, flight period and conservation status, Part I, comprising the families: Papilionidae, Pieridae, Nymphalidae (in part) and Hesperiidae describing a new species of Hypsochila (Pieridae) and a new subspecies of Yramea modesta (Nymphalidae). Boletín del Museo Nacional de Historia Natural Chile 63: 9-31.

Blanchard, E. 1852. Fauna chilena. Insectos. Orden VI. Lepidópteros. En: Gay, C (Ed.) Historia física y política de Chile: 1-112. Imprenta de Maulde et Renou, Paris.

Bartlett-Calvert, W. 1886. Catálogo de los lepidópteros Rhopalóceros i Heteróceros de Chile. Anales de la Universidad de Chile 69: 314-352.

Bartlett-Calvert, W. 1898. Catálogo revisado de los Lepidópteros de Chile. Revista chilena de Historia Natural 2: 97-101.

Boisduval, J.B.A.D. 1875. Species général des Lépidoptères Hétérocères. Tôme premier: Sphingides, Séssiides, Castnides. Libraire Encyclopédique de Roret, Paris. 568 pp.

Butler, A.G. 1877. Illustrations of typical specimens of Lepidoptera Heterocera in the collection of the British Museum. Part. I. London: Taylor \& Francis. 62 pp.

ButLer, A.G. 1881. List of butterflies collected in Chili by Thomas Edmonds, Esq. Transactions of the Entomological Society of London 1881: 449-486.

ButLer, A.G. 1882. Heterocerous Lepidoptera collected in Chili by Thomas Edmonds, Esq. Part I - Sphinges and Bombyces. Transactions of the Entomological Society of London 1882: 1-29.

Crowley, P. 1884. A note on Castnia eudesmia Gray. Proceedings of the Entomological Society of London 1884(1): i.

Elwes, H.J. 1903. The butterflies of Chile. Transactions of the Royal Entomological Society of London 51(3): 263-301.

Feisthamel, J.F. 1839. Supplement a la zoologie du voyage de la Favorite comprenant la description de lépidoptères nouveaux. Magasin de Zoologie 9: 17-26

Gazulla, P., Ruíz, F. 1928. Los insectos de la Hacienda de "Las Mercedes". Revista Chilena de Historia Natural 32: 288305.

GonzÁlez, J.M., Boone, J.H., Brilmyer, G.M., Le, D. 2010. The Giant Butterfly-moths of the Field Museum of Natural History, Chicago, with notes on the Herman Strecker collection (Lepidoptera: Castniidae). SHILAP Revista de 
lepidopterología 38(152): 385-409.

Gray, G.R. 1838. Synopsis of the species belonging to the genus Castnia. Transactions of the Entomological Society of London 2: 140-149.

Herrera, J., Etcherverry, M. 1960. Papilionidae de Chile. Apartado de la Revista universitaria (Universidad Católica de Chile) 44-45: 153-155.

Herrera, J., Atria, J. 1992. La familia Papilionidae en Chile (Lepidoptera). Acta Entomológica Chilena 17: 21-35.

Herrich-Schäffer, G.A.W. 1854. Sammlung neuer oder wenig bekannter aussereuropäischer Schmetterlinge. Regensburg, Germany. 84 pp +120 pl.

Houlbert, C. 1918. Revisión monographique de la sous-famille des Castniinae. Etudes de Lépidoptérologie Comparée 15: $1-730$.

KIRBY, W.F. 1897. A handbook to the order Lepidoptera Vol III: Butterflies (continued) - Hesperiidae, Moths Part I. Edward Lloyd Limited: London. 308 pp.

Lamas, G. 1995. A critical review of J.Y. Miller's Checklist of the Neotropical Castniidae (Lepidoptera). Revista Peruana de Entomología 37: 73-87.

Lamas, G. 2004. Papilionidae, En: Heppner, J.B. (Ed). Checklist: Atlas of Neotropical Lepidoptera Part 4A, HesperioideaPapilionoidea: 87-98. Association for Tropical Lepidoptera, Scientific Publishers, Gainesville, Florida.

LATTY, P. 1922. An account of the Castniinæ in the collection of Madame Gaston Fournier. The Annals and Magazine of Natural History; Zoology, Botany, and Geology 9: 68-86.

Miller, J.Y. 1995. Castniidae. En: Heppner, J.B. (Ed.). Checklist: Atlas of Neotropical Lepidoptera Part 2, HyblaeoideaPyraloidea-Tortricoidea: 133-137, 176-177. Association for Tropical Lepidoptera, Scientific Publishers, Gainesville, Florida.

MolinA, J.I. 1782. Saggio sulla storia naturale del Chili. Stamperia di S. Tommaso d'Aquino, Bolonia, Italia. 368 pp.

MolinA, J.I. 1788. Compendio de la Historia Geográfica, Natural y Civil del Reyno de Chile, Primera Parte. Traducción en español de Don Domingo Joseph, Madrid, España. 414 pp.

Moraes, S.D.S., Duarte, M. 2014. Phylogeny of Neotropical Castniinae (Lepidoptera: Cossoidea: Castniidae): testing the hypothesis of the mimics as a monophyletic group and implications for the arrangement of the genera. Zoological Journal of the Linnean Society 170: 362-399.

Niemeyer, H., Bustamente, R., Simonetti, J., Teillier, S., Fuentes, E. 2002. Historia natural de la Reserva Nacional Río Clarillo: un espacio para aprender ecología. Impresos Socías, Santiago, Chile. 313 pp.

Parra, L.E., Villagrán-Mella, R. 2008. Orden Lepidoptera (mariposas). En: Conama (Eds.). Biodiversidad de Chile, patrimonio y desafíos: 159-165. Santiago, Chile.

Penco, F.C. 2011. Lepidoptera Argentina: Catálogo ilustrado y comentado de las mariposas de Argentina, Parte I: Castniidae, Publicación del Autor. 40 pp.
Penco, F.C., Di Iorio, O. 2014. Lepidoptera Argentina: Catálogo ilustrado y comentado de las mariposas de Argentina, Parte VII: Papilionidae, Publicación del Autor. 88 pp.

Peña, L.E., Ugarte, A. 2006. Las Mariposas de Chile. Santiago, Chile: Editorial Universitaria. 359 pp.

Pérez-D’Angello, V. 1965. El “Catálogo de lepidópteros de Chile” de Emilio Ureta Rojas. Noticiaro mensual del Museo de Historia Natural, Santiago 104: 1-6.

Pérez-D’Angello, V. 1967 Los insectos en el "Saggio" de Molina. Noticiario Mensual del Museo Nacional de Historia Natural, Chile 124: 1-5.

Pfeiffer, L. 1915. Eine noch nicht beschriebene form von Castnia eudesmia Gray: Castnia eudesmia omissa subsp. nov. Entomologische Zeitschrift 29: 5-6.

PhiLIPPI, R.A. 1863. Metamorphosis von Castnia. Stettin Entomologisache Zeitung 24(10-12): 337-341.

PhiLIPPI, R.A. 1867. Comentario crítico sobre los animales descritos por Molina. Anales de la Universidad de Chile 29: 779.

Pyrcz, T., Ugarte, A., Boyer, P., Shapiro, A.M., Benyamini, D. 2016. An updated list of the Butterflies of Chile (Lepidoptera, Papilionoidea) including distribution, flight periods and conservation status. Part II, Subfamily Satyrinae (Nymphalidae), with the descriptions of new taxa. Boletín del Museo Nacional de Historia Natural Chile 65: 31-67.

ReED, E.C. 1877. Una monografía de las mariposas chilenas. Anales de la Universidad de Chile 49: 647-738.

Reed, E.P. 1935. La Castnia eudesmia, Gray. Revista Chilena de Historia Natural 39: 267-271.

Rothschild, W., JoRDAn, K. 1906. A revision of the American Papilios. Novitates Zoologicae 13(3): 411-752.

Silva-FigueroA, C. 1920. Revisión de los Lepidópteros Ropalóceros de Chile. Boletín del Museo Nacional de Historia Natural, Chile 11: 198-214.

Stuardo, J.R. 2007. Trascendencia del primer Saggio sulla storia naturale del Chili de J.I. Molina, su traducción, el Compendio Anónimo y el Bicentenario. Atenea 495: 83107.

Tyler, H., Brown JR, K.S., Wilson, K. 1994. Swallowtail butterflies of the Americas. A study in biological dinamics, ecological diversity, biosystematics and conservation. Scientific Publishers, Gainesville, Florida. 376 pp.

Ureta, E. 1937. Lista de Ropaloceros de Chile. Boletín del Museo Nacional de Historia Natural, Chile 16: 121-130.

UretA, E. 1955. Castnia psittacus (Molina, 1781), nueva combinación. Revista Chilena de Entomología 4: 229-231.

WALKER, F. 1854. List of the species of the lepidopterous insects in the collections of the British Museum Part. I: Lepidoptera Heterocera. London: British Museum. 762 pp.

Westwood, J.O. 1877. A monograph of the Lepidopterous genus Castnia and some allied groups. Transaction of the Linnean Society of London 2(1): 155-208.

Recibido: 30.11 .2017

Aceptado: 28.02.2018 